

\title{
Anomalies, instantons and chiral symmetry breaking at a Lifshitz point
}

\author{
loannis Bakas \\ Department of Physics, School of Applied Mathematics and Physical Sciences \\ National Technical University, 15780 Athens, Greece \\ E-mail: bakas@mail.ntua.gr
}

We give a new twist to an old-fashioned topic in quantum field theory describing violations of the chiral charge conservation of massless fermions through Adler-Bell-Jackiw anomalies in the background of instanton fields in the context of non-relativistic Lifshitz theories. The results we report here summarize in a nut-shell our earlier work on the subject found in arXiv:1103.5693 and arXiv:1110.1332. We present simple examples where index computations can be carried out explicitly focusing, in particular, to gravitational models of Lifshitz type and highlight their differences from ordinary gravity in four space-time dimensions.

Proceedings of the Corfu Summer Institute 2011 "School and Workshops on Elementary Particle Physics and Gravity"

September 4-18, 2011

Corfu, Greece 


\section{Introduction}

The axial anomalies arising upon quantization of massless fermions in a given gauge and/or metric field background are deeply connected to the analytic index of the fermion operator. The Atiyah-Singer index theorem asserts that the difference of positive and negative chirality normalizable zero modes of the Dirac operator in a given background is provided by (one-half) the integrated form of the anomalous axial current conservation law. This profound relation constitutes the backbone of our study and it can be easily established without knowing the local form of the axial anomaly. It will be discussed first for relativistic fermion theories in four space-time dimensions and then it will be generalized to non-relativistic models of Lifshitz type. Of course, the computation of the index relies on the form of the axial anomaly, which is a topological density given by the Chern-Pontryagin class of the gauge and/or metric field background. The coefficient of the anomaly turns out to be universal (it is the same for both relativistic and Lifshitz theories) in accordance with the general expectation that the axial anomaly is an infra-red phenomenon in disguise. Furthermore, if the index of the Dirac operator is non-zero, the corresponding axial charge will not be conserved in time, leading to violation of baryon and lepton number in physical processes within a given theory. These issues will be addressed in detail focusing on the similarities and the differences exhibited by relativistic and Lifshitz quantum field theories.

The results we report in the following are restricted to four-dimensional theories and they are based on our previous work on the subject $[1,2]$ to which we refer the interested reader for further details. However, the presentation we adopt here is somewhat different emphasizing more the general ideas rather than the technical details. Also, the instanton backgrounds on which the index computations will be made are described in all generality in our work on gravitational HořavaLifshitz models [3, 4]. References to other original papers can also be found there.

\section{Relativistic field theories}

First, to set up the stage, we consider the Dirac operator in a four-dimensional space-time $M_{4}$ with Euclidean signature which may also be coupled to a (generally non-Abelian) background gauge field $A_{\mu}$ via the rule of minimal substitution

$$
D_{\mu}=\partial_{\mu}+\frac{1}{8}\left[\gamma_{a}, \gamma_{b}\right] \omega_{\mu}^{a b}-i A_{\mu} .
$$

Here, $\omega_{\mu}{ }^{a b}$ are the components of the spin connection with tangent space-time indices $a, b$ and $\gamma_{a}$ are the corresponding Dirac matrices satisfying the anti-commutation relations

$$
\left[\gamma_{a}, \gamma_{b}\right]_{+}=2 \delta_{a b}
$$

In most applications, the four-dimensional metric will be taken to be of the special form

$$
d s^{2}=d t^{2}+g_{i j}(t, x) d x^{i} d x^{j}
$$

This is also appropriate for the description of Lifshitz theories satisfying the so called projectable condition, which will occupy most of our attention later. In the relativistic case, however, the space-time metric can be of general form. 
Then, the Euclidean Dirac operator and the associated $\gamma_{5}$-matrix $\gamma_{5}=-\gamma_{0} \gamma_{1} \gamma_{2} \gamma_{3}$ that anticommutes with it assume the following form in the chiral representation, respectively,

$$
i \gamma^{\mu} D_{\mu}=i\left(\begin{array}{cc}
0 & \mathscr{Q}_{-} \\
\mathscr{Q}_{+} & 0
\end{array}\right), \quad \gamma_{5}=\left(\begin{array}{cc}
1 & 0 \\
0 & -1
\end{array}\right),
$$

setting for notational convenience

$$
\mathscr{Q}_{ \pm}=\frac{\partial}{\partial t} \pm i \sigma_{I} E_{I}{ }^{i} D_{i}
$$

In writing (2.5) we use the temporal choice $A_{0}=0$ in the presence of gauge fields. We also use the Pauli matrices $\sigma_{I}$ as well as the inverse dreibeins $E_{I}{ }^{i}$ associated to the metric $g_{i j}$ in (2.3) (summation over the space indices $i$ and the tangent space indices $I$ are implicitly assumed). The $2 \times 2$ blocks $\mathscr{Q}_{ \pm}$are first-order operators mapping the two-component Weyl spinors $\Psi_{ \pm}$to $\Psi_{\mp}$ and they are mutually related by conjugation as $\left(\mathscr{Q}_{ \pm}\right)^{\dagger}=-\mathscr{Q}_{\mp}$. The massless Dirac equation $i \gamma^{\mu} D_{\mu} \Psi(t, x)=0$ acting on four-component spinors reduces to the following system of Weyl equations on $M_{4}$,

$$
\mathscr{Q}_{ \pm} \Psi_{ \pm}(t, x)=0
$$

whose number of normalizable solutions will be denoted by $n_{ \pm}$, respectively.

The index of the Dirac operator is defined as the difference between the number of positive and negative chirality zero modes, i.e.,

$$
\operatorname{Ind}(D)=n_{+}-n_{-}=\operatorname{dim} \operatorname{Ker} \mathscr{Q}_{+}-\operatorname{dim} \operatorname{Ker} \mathscr{Q}_{-}=\operatorname{Tr} \gamma_{5}
$$

The last equality is actually a tautology following from the chirality condition $\gamma_{5} \Psi_{ \pm}= \pm \Psi_{ \pm}$by taking the trace over the zero energy states in the fermion Hilbert space. One can extend the trace to the entire Hilbert space without affecting the index because the non-zero energy states are always paired and for them the difference between the two chiralities cancel. Thus, only zero energy states contribute to the index. Since the zero modes of $\mathscr{Q}_{ \pm}$are also zero modes of $\mathscr{Q}_{\mp} \mathscr{Q}_{ \pm}$, the index takes the equivalent form

$$
\operatorname{Ind}(D)=\operatorname{dim} \operatorname{Ker}\left(\mathscr{Q}_{-} \mathscr{Q}_{+}\right)-\operatorname{dim} \operatorname{Ker}\left(\mathscr{Q}_{+} \mathscr{Q}_{-}\right)=\operatorname{Tr}\left(\gamma_{5} e^{-\tau\left(i \gamma^{\mu} D_{\mu}\right)^{2}}\right) .
$$

The operators $\mathscr{Q}_{\mp} \mathscr{Q}_{ \pm}$are elliptic and they are better behaved than $\mathscr{Q}_{ \pm}$, since they are related to the square of the Dirac operator as

$$
-\left(i \gamma^{\mu} D_{\mu}\right)^{2}=\left(\begin{array}{cc}
\mathscr{Q}_{-} \mathscr{Q}_{+} & 0 \\
0 & \mathscr{Q}_{+} \mathscr{Q}_{-}
\end{array}\right) .
$$

The equality (2.8) is very useful for comparison with the axial anomaly computations. It holds for any $\tau>0$ and it provides a regulated version of $\operatorname{Tr} \gamma_{5}$. The trace is taken oven the entire fermionic Hilbert space, since the non-zero energy states cancel each other.

The massless fermion theory with Lagrangian density $\mathscr{L}=\bar{\Psi} i \gamma^{\mu} D_{\mu} \Psi$ exhibits an axial current conservation law $\nabla_{\mu} J_{5}^{\mu}(t, x)=0$, where $J_{5}^{\mu}(t, x)=\bar{\Psi} \gamma^{\mu} \gamma_{5} \Psi$. There is an invariance of the classical 
theory that can be easily found by applying Noether's procedure with respect to the chiral rotations $\delta_{\varepsilon} \Psi=i \varepsilon \gamma_{5} \Psi$. There is also an associated chiral charge

$$
Q_{5}=\int d^{3} x \sqrt{\operatorname{det} g} J_{5}^{0}(t, x)
$$

which is conserved in time under the appropriate boundary conditions at spatial infinity (typically the spatial slices are taken to be compact without boundary). Quantum mechanically, however, the situation changes drastically as there is an obstruction to the axial current conservation law, called axial anomaly. The anomaly is most conveniently described in the Euclidean domain after Wick rotation of the time coordinate and it originates from the non-invariance of the fermionic path integral measure $(\mathscr{D} \bar{\Psi})(\mathscr{D} \Psi)$ under chiral rotations. Careful investigation of the partition function shows that the variation of the action combines with the variation of the measure to produce the anomalous conservation law

$$
\nabla_{\mu} J_{5}^{\mu}(t, x)=2 \lim _{\Lambda \rightarrow \infty} \sum_{n} \varphi_{n}^{\dagger}(t, x) \gamma_{5} e^{-\left(i \gamma^{\mu} D_{\mu}\right)^{2} / \Lambda^{2}} \varphi_{n}(t, x)
$$

after introducing a cut-off $\Lambda$ to regulate the infinite sum that otherwise is ill-defined. Here, $\varphi_{n}(t, x)$ are the eigen-states of the interacting fermion operator $i \gamma^{\mu} D_{\mu}$.

Integration over space-time is combined with the sum over $n$ in (2.11) to yield the trace over the entire fermionic Hilbert space of the theory. Thus, comparison with the index formula (2.8) leads to the profound relation

$$
\operatorname{Ind}(D)=\frac{1}{2} \int_{M_{4}} d t d^{3} x \sqrt{\operatorname{det} g} \nabla_{\mu} J_{5}^{\mu}(t, x),
$$

which connects the physics of axial anomalies with the mathematical theory of Atiyah-Singer index theorem for the Dirac operator. Clearly, if the index is non-zero, the axial charge $Q_{5}$ will not be conserved, leading to baryon and lepton number violation in the theory, as

$$
\Delta Q_{5}=2 \operatorname{Ind}(D)
$$

So far there has been no explicit reference to the form of the axial anomaly in the background of gauge and/or metric fields. Likewise, there has been no reference to the Atiyah-Singer formula for computing the index of the Dirac operator analytically. This step is crucial for telling the rest of the story in physics and mathematics. Manipulating the regulated sum (2.11), it turns out that

$$
\partial_{\mu} J_{5}^{\mu}=\frac{1}{4 \pi^{2}} \operatorname{Tr}(F \wedge F), \quad \nabla_{\mu} J_{5}^{\mu}=\frac{1}{96 \pi^{2}} \operatorname{Tr}(R \wedge R)
$$

for the gauge and the gravitational field contribution to the anomalous divergence of the axial current, respectively. The obstruction to the axial current conservation law can be easily guessed in both cases. The anomaly should be a topological density built out of the field strength of the background fields, i.e., the curvature two-forms $F$ and $R_{a b}$ of the gauge and metric fields, respectively, so that the divergence of the axial current is a total derivative gauge invariant term. Furthermore, it should be odd under parity since $J_{5}^{\mu}$ is a pseudo-vector current. The only available such quantities in four space-time dimensions are the characteristic classes $\operatorname{Tr}(F \wedge F)$ and $\operatorname{Tr}(R \wedge R)$, where the 
trace is taken over the color indices of the non-Abelian gauge field (per flavor of Dirac fermions) and the tangent space-time indices of $M_{4}$, respectively. Then, it only remains to fix the coefficient of the anomaly to complete the derivation of (2.14). The outcome is the same using either physical or mathematical techniques to make sense of the regulated sum for $\operatorname{Tr} \gamma_{5}$.

Soon after the discovery of instantons in four-dimensional gauge theories and gravity, the question arose whether the index of the Dirac operator is non-zero on such topologically nontrivial backgrounds. For non-Abelian gauge fields (e.g., $S U(2)$ ) on $M_{4} \simeq S^{4}$, obtained as onepoint compactification of $\mathbb{R}^{4}$ by imposing appropriate boundary conditions on $A_{\mu}(x)$, the instanton number $k \in \mathbb{Z}$ is provided by the second Chern number

$$
k=\frac{1}{8 \pi^{2}} \int_{M_{4}} \operatorname{Tr}(F \wedge F)
$$

and, therefore, $\operatorname{Ind}(D)=k$ on such instanton backgrounds. Then, it follows that the axial charge conservation law is violated by $\Delta Q_{5}=2 k$. Likewise, on compact Riemannian manifolds without boundaries, but with non-vanishing Hirzebruch signature,

$$
\tau\left(M_{4}\right)=\frac{1}{24 \pi^{2}} \int_{M_{4}} \operatorname{Tr}(R \wedge R),
$$

the index of the Dirac operator is non-zero, $\operatorname{Ind}(D)=\tau / 8$ (recall that the signature of all compact four dimensional spin manifolds without boundaries is integer multiple of 8 ). The only gravitational instanton of this kind is $K 3$, which is a hyper-Kähler manifold with self-dual Riemann tensor whose signature is 16 and $\operatorname{Ind}(D)=2$.

In the presence of boundaries one considers bound states of the Dirac equation by imposing appropriate boundary conditions on the spinors near $\partial M_{4}$ and uses the Atiyah-Patodi-Singer (APS) index theorem to count the difference between positive and negative chirality zero modes $\left(L^{2}\right.$-index of the Dirac operator). The APS theorem is based on the integrated form of the axial anomaly with the same bulk contribution as before, but it also contains suitable boundary terms given by

$$
\operatorname{Ind}(D)=\frac{1}{192 \pi^{2}} \int_{M_{4}} \operatorname{Tr}(R \wedge R)-\frac{1}{192 \pi^{2}} \int_{\partial M_{4}} \operatorname{Tr}(\theta \wedge R)-\frac{1}{2} \eta_{\mathrm{D}}\left(\partial M_{4}\right)
$$

The first boundary term involves the Chern-Simons secondary characteristic class written in terms of the second fundamental form $\theta$, which accounts for the possible deviation of the space-time metric $d s^{2}=d t^{2}+g_{i j}(t, x) d x^{i} d x^{j}$ from cross-product form at the boundary; as such it is a higher derivative analogue of the Gibbons-Hawking-York boundary term encountered in general relativity. The second boundary term is non-local and it is provided by the $\eta$-invariant of the tangential part of the Dirac operator restricted to boundary; as such it counts the spectral asymmetry between positive and negative chirality modes of the corresponding three-dimensional Dirac operator on $\partial M_{4}$ and it is made rigorous using zeta-function regularization.

When $M_{4}=I \times \Sigma_{3}$ with a compact three-manifold $\Sigma_{3}$ without boundaries (e.g., $\Sigma_{3} \simeq S^{3}$ ), the index of the Dirac operator on $M_{4}$ can be calculated in practice by spectral flow methods. $\operatorname{Ind}(D)$ is provided by the net number of level crossings that occur in the spectrum of the three-dimensional Dirac operator on $\Sigma_{3}$,

$$
\operatorname{Ind}(D)=\Delta S\left(\Sigma_{3}\right)
$$


as the metric $g_{i j}(t, x)$ deforms from one end of the time interval $I$ to the other; recall that the $\eta$-invariant jumps by \pm 2 units when an eigen-value crosses from negative to positive values or conversely, and, therefore, each level crossing contributes \pm 1 units to the index. Formula (2.18) and its generalization to Lifshitz theories is very useful for the applications. In those cases that the complete spectrum of the Dirac operator on $\Sigma_{3}$ can be found and the spectral flow can be studied explicitly, the index of the four-dimensional fermion operator on $I \times \Sigma_{3}$ can be computed in closed form.

It is well known that all non-compact instanton solutions of Einstein gravity can not support extreme geometric deformations on $\Sigma_{3}$ that are capable to induce level crossing, and, hence, $\operatorname{Ind}(D)=0$. Prime examples of this kind are provided by the Taub-NUT and Eguchi-Hanson instantons which admit complete metrics (2.3) with self-dual Riemann curvature tensor on $I \times \Sigma_{3}$ with $I$ being the semi-infinite real line of proper time $t$ and $\Sigma_{3}$ is $S^{3}$ and $S^{3} / \mathbb{Z}_{2}$, respectively, endowed with homogeneous and partially isotropic geometries with $S U(2) \times U(1)$ isometry group. It can be explicitly seen in those cases that the individual terms contributing to the index (2.17) cancel against each other, as required on general grounds based on Lichnerowicz's theorem: non-compact four-metrics with non-negative Ricci scalar curvature admit no bound state solutions of the Dirac equation (if such states existed, they would be covariantly constant, and, hence, non-normalizable leading to contradiction). Thus, it is not possible to have chiral symmetry breaking induced by gravitational instantons of topology $I \times \Sigma_{3}$ in ordinary Einstein-Dirac theory.

\section{Lifshitz field theories}

Next, we extend the scope of our discussion to Lifshitz fermion theories with anisotropy scaling exponent $z=2 \alpha+1$ by considering the non-relativistic analogue of the Dirac operator

$$
i \gamma^{\mu} \mathscr{D}_{\mu}=i \gamma^{0} D_{0}+\frac{1}{2} i \gamma^{i}\left[D_{i}\left(-D_{k} D^{k}+M^{2}\right)^{\alpha}+\left(-D_{k} D^{k}+M^{2}\right)^{\alpha} D_{i}\right]
$$

in the presence of background gauge and/or metric fields. Here, $M$ is an arbitrary mass scale that is introduced for convenience to extrapolate between the Dirac and Lifshitz fermion models. The Lifshitz operator acts on four-component spinors $\Psi(t, x)$ and $D_{0}$ and $D_{i}$ are the time and space components of the ordinary covariant derivative (2.1) that provides the minimal coupling to the background fields. Then, the massless Lifshitz fermion theory in $3+1$ space-time dimensions is defined by the Lagrangian density $\bar{\Psi} i \gamma^{\mu} \mathscr{D}_{\mu} \Psi$ and gives rise to the axial current conservation law $\nabla_{\mu} J_{5}^{\mu}(t, x)=0$, as in the relativistic case. The spatial components of the axial current are different from the relativistic case, since they involve a number of derivatives that depend upon $z$, whereas the time component $J_{5}^{0}(t, x)$, and, hence, the axial charge $Q_{5}$ is the same.

The axial symmetry is broken quantum mechanically and explicit computation shows that the anomalous term in the divergence of the axial current is identical to the relativistic case for the gauge and/or the metric field couplings, as in equation (2.14). More precisely, the anomalous divergence of the axial current in the presence of gauge fields is

$$
\partial_{\mu} J_{5}^{\mu}=\frac{1}{4 \pi^{2}} \varepsilon^{0 i j k} \operatorname{Tr}\left(F_{0 i} F_{j k}\right)=\frac{1}{4 \pi^{2}} \operatorname{Tr}(F \wedge F),
$$


whereas the metric field contribution to the axial anomaly turns out to be

$$
\nabla_{\mu} J_{5}^{\mu}=-\frac{1}{96 \pi^{2}} \varepsilon^{0 i j k} R^{a b}{ }_{0 i} R_{a b j k}=\frac{1}{96 \pi^{2}} \operatorname{Tr}(R \wedge R) .
$$

Here, we write the result in $3+1$ terms using the electric and magnetic components of the corresponding curvature 2-forms and then recast it in the form (2.14). The calculation is performed in the Euclidean domain by analytic continuation of the space-time foliation used for the formulation of Lifshitz models. It is also implicitly assumed that the space-time metric is restricted to the projectable case, meaning that the lapse and shift functions in the ADM decomposition of the metric are taken to be 1 and 0 , respectively, so that its analytic continuation is given by (2.3) in proper time $x^{0}=t$.

The arguments leading to the general relation (2.12) between the index of the fermion operator and the axial anomaly generalize easily to the Lifshitz operator (3.1). One simply has to consider the higher derivative analogue of the operators $\mathscr{Q}_{ \pm}$shown in (2.5) by replacing $D_{i}$ with $\left[D_{i}\left(-D_{k} D^{k}+M^{2}\right)^{\alpha}+\left(-D_{k} D^{k}+M^{2}\right)^{\alpha} D_{i}\right] / 2$ and also replace the regulator $\exp \left[-\left(i \gamma^{\mu} D_{\mu}\right)^{2} / \Lambda^{2}\right]$ by $\exp \left[-\left(i \gamma^{\mu} \mathscr{D}_{\mu}\right)^{2} / \Lambda^{2 z}\right]$ in the sum (2.11) which is now taken oven the eigen-states $\varphi_{n}(t, x)$ of the interacting Lifshitz fermion operator $i \gamma^{\mu} \mathscr{D}_{\mu}$. As a result, the index of the Lifshitz fermion operator (3.1) is equal to the index of the Dirac operator for all values of the anisotropy exponent $z$,

$$
\operatorname{Ind}(\mathscr{D})=\operatorname{Ind}(D) \text {. }
$$

In space-times with boundaries the Atiyah-Patodi-Singer index theorem for the Lifshitz fermion operator assumes the same form as in (2.17). The $\eta$-invariant is now referring to the tangential part of the Lifshitz operator restricted to the boundary $\partial M_{4}$, which turns out to be equal to the $\eta$ invariant of the corresponding three-dimensional Dirac operator. Thus, relation (3.4) is universal, as it extends to all space-times with or without boundaries. If the index does not vanish, the axial charge will not be conserved in time, i.e., $\Delta Q_{5}=2 \operatorname{Ind}(\mathscr{D})$, leading to baryon and lepton number violation as before. It will be seen shortly that chiral symmetry breaking effects in non-relativistic theories of Lifshitz type are common practice.

Anomalies, instantons and chiral symmetry breaking at a Lifshitz point are closely interrelated as in the relativistic case. The task is to find solutions of Lifshitz theories that allow for violation of chiral charge conservation and then compare the results to relativistic field theories. Here, we provide a brief account of bosonic Lifshitz theories and review their instanton solutions, following our earlier work on the subject [3,4]. In general, they provide classical backgrounds for Lifshitz fermion propagation in $3+1$ space-time dimensions. Special emphasis is placed on gravitational theories of Lifshitz type (in the so called Hořava-Lifshitz gravity) coupled to Lifshitz fermion models. Then, ignoring the backreaction of fermions to the gravitational instanton backgrounds, we find that - unlike ordinary gravity - non-conservation of $Q_{5}$ becomes possible for a certain range of the gravitational coupling parameters. We provide some simple solutions that realize this novel possibility and give a qualitative interpretation of its origin. The case of Lifshitz gauge field theories appears to be conceptually simpler and looks easier for comparison with the relativistic case, but its instanton solutions are not explicitly known to this day; it is an open problem for future study which we hope to address elsewhere in detail.

The bosonic Lifshitz field theories in $3+1$ space-time dimensions resemble point particle systems with configuration space $\mathscr{C}$ and local coordinates $q_{I}$ that correspond to Euclidean relativistic 
fields in three spatial dimensions. Thus, $\mathscr{C}$ is the infinite dimensional space of all scalar, vector or more generally tensor fields on a Riemannian manifold $\Sigma_{3}$, i.e., $q_{I}=\varphi(x), A_{i}(x)$ or $g_{i j}(x)$ etc, which will be called superspace in all cases. These field theories are non-relativistic models with anisotropic scaling in space and time $x \rightarrow a x$ and $t \rightarrow a^{z} t$ with exponent $z$ which is provided by the order of the classical equations of motion of the relativistic fields $q_{I}$ defined on $\Sigma_{3}$. Their action is often taken to be of the form

$$
S=\frac{1}{2} \int d t \sum_{I, J}\left(\frac{d q_{I}}{d t} \mathscr{O}^{I J} \frac{d q_{J}}{d t}-\frac{\partial W}{\partial q_{I}} \mathscr{O}_{I J} \frac{\partial W}{\partial q_{J}}\right)
$$

assuming that the potential term is derivable from a superpotential $W[q]$ using the metric $\mathscr{O}^{I J}$ and its inverse $\mathscr{O}_{I J}$ on the superspace $\mathscr{C}$. This class of Lifshitz models are said to satisfy the detailed balance condition with local superpotential functional $W$ being the action of a suitably chosen relativistic field theory on $\Sigma_{3}$. Of course, one may deviate from detailed balance by having additional terms in the potential that cannot be casted in the form (3.5) using a local superpotential functional, but such generalizations will not be in focus here. In all cases, the Lifshitz theories admit an effective point particle description in superspace that proves useful in many respects.

Next, we consider instanton solutions of Lifshitz theories on $\mathbb{R} \times \Sigma_{3}$, assuming for simplicity that the metric in superspace is positive definite (it can also become degenerate in some important cases that will be discussed later). We first note that the minima of the potential provide static solutions of the equations of motion following from (3.5); they are configurations that satisfy the classical equations of motion $\partial W / \partial q_{I}=0$ of the underlying relativistic field theory defined by the action $W$ on $\Sigma_{3}$ and they are all degenerate with zero energy. Then, the instantons are defined as extrema of the Euclidean action derived from (3.5) by Wick rotation $t \rightarrow i t$ (equivalently by inverting the potential) that interpolate smoothly between different degenerate vacua of the effective point particle system as $t$ extends all the way from $-\infty$ to $+\infty$. By completing the square, as usual, it can be easily seen that the instantons satisfy the system of first-order equations in time

$$
\frac{d q_{I}}{d t}= \pm \mathscr{O}_{I J} \frac{\partial W}{\partial q_{J}}
$$

and their action equals $S_{\text {instanton }}=|\Delta W| \equiv|W(t=+\infty)-W(t=-\infty)|$. The two choices of sign in (3.6) correspond to instanton and anti-instanton configurations.

Thus, the instantons of Lifshitz theories (with detailed balance) are eternal solutions of the gradient flow equations (3.6) derived from the superpotential functional $W$ and their action is finite, as required on general grounds. Yet explicit solutions of the gradient flow equations can not be easily obtained unless additional symmetries are imposed on the fields, leading to consistent minisuperspace truncations of the configuration space $\mathscr{C}$. Otherwise, only qualitative features of the solutions can be studied, in general, at least in those cases that the mathematical tools of geometric analysis are powerful enough to explore the problem of long-time existence against the possible formation of singularities along the flow lines. In gravitational theories of Lifshitz type the defining relations (3.6) are nothing else but geometric flows for the metrics on $\Sigma_{3}$. Specific examples and explicit solutions will be described later and compared to the instantons of Einstein gravity. Note for completeness that if we were considering Lifshitz theories without detailed balance, instanton solutions would be much more difficult to find, if they existed at all as finite Euclidean action configurations. 
Specializing to Hořava-Lifshitz gravity, we consider $(3+1)$-dimensional space-times $M_{4} \simeq$ $\mathbb{R} \times \Sigma_{3}$ endowed with Lorentzian metrics $d s^{2}=-d t^{2}+g_{i j}(t, x) d x^{i} d x^{j}$ (the so called projectable case) and write down the following action in canonical form,

$$
S=\frac{1}{2} \int_{M_{4}} d t d^{3} x \sqrt{\operatorname{det} g}\left[\left(\frac{\partial g_{i j}}{\partial t}\right) \mathscr{G}^{i j k l}\left(\frac{\partial g_{i j}}{\partial t}\right)-\left(\frac{1}{2 \sqrt{\operatorname{detg}}} \frac{\delta W}{\delta g_{i j}}\right) \mathscr{G}_{i j k l}\left(\frac{1}{2 \sqrt{\operatorname{det} g}} \frac{\delta W}{\delta g_{k l}}\right)\right],
$$

where

$$
\mathscr{G}^{i j k l}=\frac{1}{2}\left(g^{i k} g^{j l}+g^{i l} g^{j k}\right)-\lambda g^{i j} g^{k l}
$$

is the ( $\lambda$-deformed) DeWitt metric in superspace $\mathscr{C}$ consisting of all Riemannian metrics on $\Sigma_{3}$ and $\mathscr{G}_{i j k l}$ is its inverse. We also choose as superpotential functional $W[g]$ the action of topologically massive gravity on the three-manifold $\Sigma_{3}$,

$$
W_{\mathrm{TMG}}[g]=\frac{2}{\kappa_{\mathrm{W}}^{2}} \int_{\Sigma_{3}} d^{3} x \sqrt{\operatorname{detg}}\left(R-2 \Lambda_{\mathrm{W}}\right)+\frac{1}{\omega} W_{\mathrm{CS}}[g],
$$

where

$$
W_{\mathrm{CS}}[g]=\int_{\Sigma_{3}} d^{3} x \sqrt{\operatorname{detg}} \varepsilon^{i j k} \Gamma_{i m}^{l}\left(\partial_{j} \Gamma_{l k}^{m}+\frac{2}{3} \Gamma_{j n}^{m} \Gamma_{k l}^{n}\right)
$$

is the gravitational Chern-Simons action, which is conveniently written here in terms of the Christoffel symbols of the metric $g$ on $\Sigma_{3}$. The resulting non-relativistic gravitational theory (3.7) in $3+1$ dimensions exhibits anisotropic scaling $z=3$ which reduces to $z=2$ only when the gravitational Chern-Simons term is absent.

The essential feature of topologically massive gravity is the presence of an adjustable scale that supplies the mass to one of the two helicity gravitons in the weak field approximation of the theory around flat space; the other helicity graviton remains massless and the two are interrelated by orientation reversing transformations on $\Sigma_{3}$ that flip the sign of $\omega$. The associated range of gravitational interactions in three dimensions is given by the relative ratio of the three-dimensional gravitational constant to the Chern-Simons coupling, $\kappa_{\mathrm{w}}^{2} /|\omega|$, which will play role later in the formulation of a geometric criterion for having chiral symmetry breaking effects by gravitational instantons in the associated $(3+1)$-dimensional Hořava-Lifshitz gravity. Further generalizations arise by adding quadratic (or higher) curvature terms to $W$, as in three-dimensional new massive gravity (and generalizations thereof), which lead to $(3+1)$-dimensional Hořava-Lifshitz models with higher anisotropy scaling exponent $z$. In those cases, the three-dimensional gravitons remain massive (although the masses of the two helicity states need not be the same) leading to similar phenomena as with $W_{\mathrm{TMG}}$; such generalizations will not be addressed here at all to simplify the presentation.

The instanton equation (3.6) specializes to the Ricci-Cotton flow. This is a third order equation derived from $W_{\mathrm{TMG}}[g]$ as gradient flow for the metric $g_{i j}$ on $\Sigma_{3}$. The flow lines depend on the superspace parameter $\lambda$, but the fixed points, which are classical solutions of three-dimensional topological massive gravity, do not depend on it for general values of $\lambda$. Here, we only consider the spacial case $\lambda=1 / 3$, so that the defining equation of gravitational instantons takes the form

$$
\partial_{t} g_{i j}=\mp \frac{1}{\kappa_{\mathrm{w}}^{2}}\left(R_{i j}-\frac{1}{3} R g_{i j}\right) \pm \frac{1}{\omega} C_{i j}
$$


where $R_{i j}$ is the Ricci curvature tensor of $g$ and $C_{i j}$ its Cotton tensor. The driving curvature terms that arise in this case are traceless. This, in turn, implies that the fixed points have undetermined Ricci scalar curvature $R$, which can vary from one fixed point to the other. Said differently, we are considering a unimodular version of three-dimensional topological massive gravity in which the cosmological constant $\Lambda_{\mathrm{w}}$ has the interpretation of an integration constant that can assume arbitrary values. This restriction is necessary for having level crossing, and, hence, $\Delta Q_{5} \neq 0$ as $t$ varies from $-\infty$ to $+\infty$ in the corresponding gravitational instanton background. Otherwise, if $\lambda \neq 1 / 3$, the two end-points of the instanton will have the same curvature $R\left(=6 \Lambda_{\mathrm{w}}\right)$ forbidding any net level crossing to occur in the theory.

More technically speaking, the metric in superspace takes the following form at $\lambda=1 / 3$,

$$
\mathscr{G}^{i j k l}=\frac{1}{2}\left(g^{i k} g^{j l}+g^{i l} g^{j k}\right)-\frac{1}{3} g^{i j} g^{k l},
$$

and as such it projects any symmetric two-tensor to its traceless part. Then, the inverse metric in superspace becomes ill-defined. Instead, one defines

$$
\mathscr{G}_{i j k l}=\frac{1}{2}\left(g_{i k} g_{j l}+g_{i l} g_{j k}\right)-\frac{1}{3} g_{i j} g_{k l},
$$

which follows formally from the inverse metric as $\lambda \rightarrow \pm \infty$ and it also projects any symmetric twotensor to its traceless part. The two quantities are simply related to each other by the generalized orthonormality condition

$$
\mathscr{G}^{i j k l} \mathscr{G}_{k l m n}=\frac{1}{2}\left(\delta_{m}^{i} \delta_{n}^{j}+\delta_{n}^{i} \delta_{m}^{j}\right)-\frac{1}{3} g^{i j} g_{m n}
$$

that follows from the standard one by subtracting the trace part for consistency of the projection. As a result, the conformal factor of the metric decouples from the dynamics and the Ricci-Cotton flow (3.11) preserves the volume of $\operatorname{space} \operatorname{Vol}\left(\Sigma^{3}\right)$. This is precisely the class of models that allow for violations of chiral charge conservation by gravitational instanton effects in Hořava-Lifshitz gravity.

Our next task is to consider simple examples that illustrate the situation and derive the necessary and sufficient conditions on the parameters of the theory for having chiral symmetry breaking. We consider the simple case of Bianchi IX homogeneous geometries on $\Sigma_{3}=S^{3}$ with isometry group $S U(2) \times U(1)$, known as Berger spheres,

$$
d s^{2}=\gamma(t)\left[\left(\sigma^{1}\right)^{2}+\left(\sigma^{2}\right)^{2}+\delta^{2}(t)\left(\sigma^{3}\right)^{2}\right],
$$

which provide consistent mini-superspace reduction of the Ricci-Cotton flow to an ordinary differential equation. Here, $\sigma^{I}$ are the left-invariant 1 -forms of $S U(2)$ satisfying

$$
d \sigma^{I}+\frac{1}{2} \varepsilon_{J K}^{I} \sigma^{J} \wedge \sigma^{K}=0
$$

and $\delta \in[0, \infty)$ is a parameter measuring the anisotropy of the model. Instanton solutions of HořavaLifshitz gravity can be explicitly constructed in this case and then compared to the analogous solutions (Eguchi-Hanson and Taub-NUT) of Euclidean Einstein gravity. Homogeneous solutions 
with higher degree of anisotropy can also be studied by relaxing the additional $U(1)$ isometry (axial symmetry) of the 3-sphere, but they will not be discussed here as they introduce unnecessary technical complications.

The normalized Ricci-Cotton flow (3.11) admits the round metric with $\delta=1$ as fixed point, but it also admits a second fixed point within the class of Berger sphere metrics provided that $\omega<0$ (for a given choice of orientation of $S^{3}$ ) with

$$
\frac{1}{3}+\frac{\kappa_{\mathrm{w}}^{2} \delta}{2 \omega \sqrt{\gamma}}=0 .
$$

The presence of two fixed points is prerequisite for the existence of instantons. There is a smooth flow line interpolating between these two fixed points, but the precise form of the solution will not be important in the following. All it matters here is the variation of the 3-curvature

$$
R=\frac{1}{2 \gamma}\left(4-\delta^{2}\right)
$$

as one moves from one fixed point to the other and the ability to induce level crossing by changing the shape of $S^{3}$, whereas the volume $\operatorname{Vol}\left(S^{3}\right)=16 \pi^{2} \delta \gamma^{3 / 2}$ remains fixed throughout the evolution. Fortunately, the computation of the index of the Dirac-Lifshitz operator is a tractable problem in the background of gravitational instanton solutions with $S U(2) \times U(1)$ isometry.

Let $\zeta$ denote the eigen-values of the three-dimensional Dirac operator $i \gamma^{i} D_{i}$ on a Berger sphere, which can be determined in closed form together with their multiplicities. They split into positive and negative eigen-values that depend upon $\delta$. Zero modes (often called harmonic spinors) also arise for special values of $\delta \geq 4$,

$$
\delta^{2}=2 \sqrt{4 p q \delta^{2}+(p-q)^{2}}, \quad(p, q) \in \mathbb{N}^{2}
$$

with multiplicities $p+q$. Setting $p=q=1$ it follows that the first zero modes of $i \gamma^{i} D_{i}$ arise when $\delta=4$, which is the critical value of the anisotropy parameter for inducing level crossing by varying $\delta$. Comparison with equation (3.18) shows that the curvature of the Berger sphere should be sufficiently negative to allow for the occurrence of zero modes and subsequently for level crossing as $\delta>4$. This is also consistent with Lichnerowicz's theorem for the three-dimensional Dirac operator that requires negative curvature for the existence of harmonic spinors.

Extending the discussion to the three-dimensional Dirac-Lifshitz operator $i \gamma^{i} \mathscr{D}_{i}$, we note that its eigen-values $Z$ on Berger spheres are simply expressed in terms of the eigen-values $\zeta$ of the corresponding Dirac operator as

$$
Z=\zeta\left(\zeta^{2}+\frac{1}{8 \gamma}\left(\delta^{2}-4\right)+M^{2}\right)^{\alpha}
$$

It turns out that $Z$ have the same sign as $\zeta$ for all values of $\delta$, and, hence, level crossing occurs at the values of $\delta$ given by (3.19) as before. Also, since the multiplicity of the eigen-values of $Z$ is the same as $\zeta$, the number of modes that undergoes level crossing, $\Delta S\left(S^{3}\right)$, is the same for both operators. This is consistent with the fact that the index of the four-dimensional Dirac-Lifshitz operator is the same as the index of the Dirac operator on all such geometrical backgrounds $\mathbb{R} \times S^{3}$, i.e., $\operatorname{Ind}(\mathscr{D})=\operatorname{Ind}(D)$, and which can be computed by spectral flow methods via equation (2.18). 
The instanton of $z=3$ Hořava-Lifshitz gravity with $S U(2) \times U(1)$ isometry that interpolates smoothly between the round sphere and the Berger sphere with $\delta$ given by (3.17) leads to violation of chiral charge conservation provided that the volume of space (which remains fixed for all $t$ ) is bounded from below as

$$
\operatorname{Vol}\left(S^{3}\right)=16 \pi^{2} \delta \gamma^{3 / 2}=54 \pi^{2} \delta^{4}\left(-\frac{\kappa_{\mathrm{W}}^{2}}{\omega}\right)^{3}>13824 \pi^{2}\left(-\frac{\kappa_{\mathrm{W}}^{2}}{\omega}\right)^{3}
$$

letting $\delta>4$ in the final step. Thus, chiral symmetry breaking becomes possible when the mean radius of space is sufficiently larger than the range of interaction $\sim \kappa_{\mathrm{w}}^{2} /|\omega|$ in topologically massive gravity, which is associated to the superpotential functional $W[g]$. This provides a qualitative criterion for chiral symmetry breaking in Hořava-Lifshitz gravity that satisfies the detailed balance condition and which generalizes beyond the simple example we have considered here. $\Delta Q_{5}$ is given by the number of modes (including their multiplicities) that have undergone level crossing.

Comparison to Einstein gravity reveals that novel phenomena become possible in non-relativistic gravitational theories leading to fermion number violation. According to a scenario, such theories are thought to provide the ultra-violet completion of gravity sacrificing relativistic invariance for power counting renormalizability at very high energies. The phase of Hořava-Lifshitz gravity arising at $\lambda=1 / 3$ seems to be more appropriate in this context although the flow to ordinary gravity in the infra-red regime and the emergence of relativistic invariance as low energy phenomenon has not been made quantitative to this day. The asymptotic safety programme for gravity seems to provide a promising framework to address this fundamental issue in future studies.

\section{Conclusions}

We outlined the general relation between anomalies, instantons and chiral symmetry breaking in relativistic and Lifshitz field theories. The quantum anomaly of the axial current conservation law of massless fermions is independent of the anisotropy scaling parameter and coincides with the result obtained for the relativistic case in the background of gauge and metric fields. Likewise, the index of the Dirac-Lifshitz operator is universal given by the integrated form of the axial anomaly. This is in agreement with the infra-red nature of the axial anomaly, which is inert to higher derivative terms that become relevant in the ultra-violet regime.

The difference between relativistic and Lifshitz field theories lies in the ability of their instantons to affect the conservation law of chiral charge. The main result in this context is the construction of simple instanton solutions of gravitational Lifshitz theories and their use to induce chiral symmetry breaking for certain range of the couplings, leading to baryon and lepton number violation triggered by gravity. This novel possibility does not arise in general relativity. It remains to construct instanton solutions of Lifshitz gauge theories and examine their effect on chiral symmetry breaking in comparison to ordinary gauge theories.

\section{Acknowledgments}

I thank the conference organizers for their kind invitation to give an account of this work in an exciting scientific environment as well as for the partial financial support. 


\section{References}

[1] I. Bakas and D. Lüst, Axial anomalies of Lifshitz fermions, Fortschr. Phys. 59 (2011) 937 [arXiv:1103.5693 [hep-th]].

[2] I. Bakas, More on axial anomalies of Lifshitz fermions, Fortschr. Phys. 60 (2012) 224 [arXiv:1110.1332 [hep-th]].

[3] I. Bakas, F. Bourliot, D. Lüst and M. Petropoulos, Geometric flows in Hořava-Lifshitz gravity, JHEP 1004 (2010) 131 [arXiv:1002.0062 [hep-th]].

[4] I. Bakas, Gradient flows and instantons at a Lifshitz point, J. Phys. Conf. Ser. 283 (2011) 012004 [arXiv:1009.6173 [hep-th]]. 